\title{
Sugaring-out extraction of erythromycin from fermentation broth
}

\author{
Sharayu Moharkar and Pradip Babanrao Dhamole ${ }^{\dagger}$ \\ Department of Chemical Engineering, Visvesvaraya National Institute of Technology, Nagpur, Maharashtra India \\ (Received 17 June 2020 • Revised 14 September 2020 • Accepted 18 September 2020)
}

\begin{abstract}
This study reports the sugaring-out extraction of erythromycin from fermentation broth using acetonitrile $(\mathrm{ACN})$ as solvent and glucose as a mass separating agent. Different process parameters--glucose concentration, temperature, $\mathrm{ACN} /$ water ratio and $\mathrm{pH}$--were optimized to achieve maximum extraction of erythromycin. $88 \%(\mathrm{w} / \mathrm{w})$ of erythromycin was extracted from the model system with following optimized conditions: glucose $156.3 \mathrm{~g} / \mathrm{L}$; temperature $4{ }^{\circ} \mathrm{C}$; ACN/water ratio 1 and $\mathrm{pH}$ 8.3. Further, the effect of typical fermentation media components (starch, soybean flour, $\mathrm{CaCO}_{3}, \mathrm{NaCl}$ and $\left.\left(\mathrm{NH}_{4}\right)_{2} \mathrm{SO}_{4}\right)$ on sugaring out extraction of erythromycin was also investigated. Starch, soybean flour and $\mathrm{CaCO}_{3}$ were observed to affect erythromycin extraction only at higher concentration. Removal of suspended solids from simulated as well as real broth prior to extraction enhanced the extraction efficiency (from $72 \%$ to $87 \%$ ). Sugaring out extraction of erythromycin was found to be more effective than salting out extraction. Also, higher partition coefficient was achieved in the present work than other reported methods using carbohydrates as mass separating agent. Further, it was found that the antimicrobial activity of erythromycin was preserved during sugaring out extraction of erythromycin.
\end{abstract}

Keywords: Erythromycin, Downstream Process, Sugaring Out, ATPS, Bioseparation

\section{INTRODUCTION}

Antibiotics have made it possible to treat many deadly diseases. Antibiotics such as tetracycline and streptomycin have potential to be used against the plague, which still poses a potential threat to human health [1]. Also, anti-leprosy antibiotics such as sulfonamides and phenicol have claimed to be beneficial for the treatment of tuberculosis [2]. Erythromycin possesses anti-inflammatory activity which is used for the treatment of airborne diseases such as asthma and panbronchiolitis and skin diseases such as acne vulgaris [3]. Some authors have claimed that use of azithromycin (a semisynthetic derivative of erythromycin) alongside hydroxychloroquinone induces anti-viral response in case of acute respiratory syndrome SARS-CoV-2. This major finding might be useful to restrict spread of coronavirus disease $[4,5]$.

Annual consumption of some of the widely used antibiotics like erythromycin is 4,000 tons [6]. Erythromycin, which is produced by fermentation in aqueous environment, has a low titre $(8-10 \mathrm{~g} / \mathrm{L})$ [6-8]. Presence of several impurities (proteinaceous matter, polysaccharides, salts, metabolites) make separation of erythromycin difficult and expensive $[8,9]$. Downstream processing of erythromycin needs a series of unit operations that result in low yield. Typically used separation processes involve one or more of the following operations: solvent extraction [10], reverse micellar [9], ion exchange [11], membrane filtration [12,13] and crystallization [14]. Solvent extraction is by far the most widely used method for erythromycin extraction from aqueous solutions. Amongst the various solvents explored, butyl acetate is extensively used as an extractant in

\footnotetext{
${ }^{\dagger}$ To whom correspondence should be addressed.

E-mail: pdhamole@che.vnit.ac.in,pradipdhamole@gmail.com

Copyright by The Korean Institute of Chemical Engineers.
}

industry for extraction of erythromycin and other antibiotics [1517]. One-third of the total downstream processing cost is due to butyl acetate. Also, butyl acetate forms a stable emulsion and takes long time ( $35 \mathrm{hrs)}$ for phase separation to take place. In addition, it has a high boiling point, which makes the recovery process energy intensive $[10,14]$.

Le et al. investigated salting out extraction of erythromycin from fermentation broth using acetonitrile $(\mathrm{ACN})$ as a solvent and $\mathrm{NaCl}$ as a mass separating agent [14]. Salting out extraction of erythromycin yielded high extraction efficiency $(>98.5 \%)$ with rapid phase separation and no stable emulsion. ACN was found to be an ideal solvent with a high partition coefficient of 60 [9]. The shortcomings of salting-out include recovery of salts, unwanted reactions, and destructive effects on some biological products $[18,19]$. Corrosion of equipment was another obstacle for salting out applications [20]. Besides, some salts (e.g., $\mathrm{K}_{2} \mathrm{HPO}_{4}$ ) may alter the $\mathrm{pH}$ and damage the product [21].

Wang et al. reported a new method wherein sugar (monosaccharide and disaccharides) is used as a mass separating agent [22, 23]. Detailed phase separation conditions for acetonitrile, water, and glucose system were studied [24]. In the last few years sugaring-out has been extensively used for separation of various products which include 2,3 butanediol [25], lactic acid [26], acetoin [27], succinic acid [28,29], antibiotics [30,31], proteins [32], phenolics $[33,34]$ and even in analysis of some compounds [35,36]. Glucose results in better separation and hence is used in most of the studies on sugaring out extraction [22-29]. One of the limitations in salting-out has been the recovery of salts from the bottom phase, which has been addressed in sugaring out. Dai et al. and Yan et al. have re-used the bottom phase containing a large amount of sugars for fermentation after removal of residual solvent [25-27]. Also, the solvent from the top phase was recovered using vacuum distil- 
lation and the solute was crystallized [29]. Besides, sugaring-out has several other advantages, such as rapid phase separation, works over a wide temperature range $\left(1-30^{\circ} \mathrm{C}\right)$ without changing environment conditions (e.g., $\mathrm{pH}$ ), and minimizing the corrosion problem [24,34].

Thus, stable emulsion formation, recovery of salt, change in environment conditions and loss of solvent are major issues in erythromycin extraction. In view of these limitations, this work was undertaken to investigate the 'sugaring-out extraction' of erythromycin from aqueous solution (simulated broth and real broth). The present work gives a novel approach for the extraction of erythromycin with respect to above-mentioned aspects. The operating conditions were optimized to achieve maximum erythromycin extraction using a model system. Earlier studies with sugaring-out showed that temperature, glucose concentration, and ACN/water ratio affect sugaring out $[24,34]$. Further, $\mathrm{pH}$ also influences the extraction of erythromycin [14]. Hence, these four parameters were optimized using Box-Behnken design (BBD) to achieve maximum extraction and the range was decided based on reported studies. At the end of fermentation, some media components may remain unutilized and hence, the effect of media components (specific and combined) on erythromycin extraction was studied. Sugaring-out extraction of erythromycin was compared with salting-out extraction. The impact of sugaring out on antimicrobial activity of erythromycin was investigated as well.

\section{MATERIALS AND METHODS}

\section{Chemicals}

Erythromycin used as a standard reference was purchased from Century Pharmaceuticals, Vadodara, Gujarat (India). HPLC grade ACN, glucose, phosphoric acid, and methanol were purchased from MERCK. Erythromycin producing strain, Sachharopolyspora erythrea (MTCC NO. 1103) was from IMTECH, Chandigarh (India). Bacteria used for antimicrobial activity test, Bacillus pumilus (NCIM NO. 2327) was bought from NCL, Pune (India).

\section{Sugaring-out Extraction of Erythromycin}

Dhamole et al. studied the phase diagram for sugaring-out in detail [24]. This formed the basis for the selection of sugaring out conditions. Aqueous glucose solution and ACN containing $10 \mathrm{~g} / \mathrm{L}$ of erythromycin were taken in the required ratio in a $15 \mathrm{~mL}$ capped graduated test tube. The mixture was then shaken thoroughly at room temperature and kept in a circulating water bath chiller (Polyscience) to maintain the temperature. After phase separation, the volume of the upper phase (ACN-rich) and the lower phase (waterrich) was recorded. Samples from both the phases were collected for further analysis.

\section{Salting Out Extraction of Erythromycin}

Le et al. explored salting-out extraction of erythromycin from fermentation broth. The optimum conditions $(\mathrm{NaCl} 10 \% \mathrm{w} / \mathrm{w}$, $\mathrm{ACN} /$ water ratio 1 , temperature $25^{\circ} \mathrm{C}$, and $\mathrm{pH} 10$ ) reported by Le et al. were used for salting-out extraction of erythromycin [14]. Volumes of the upper phase and lower phase were noted and samples were collected from both the phases for erythromycin analysis.

\section{Optimization of Process Parameters: Experimental Design}

Process parameters for sugaring out extraction of erythromycin were optimized for a model system following response surface meth-
Table 1. Box Behnken Design arrangement and response $\left(X_{1}\right.$ : Glucose concentration, $\mathrm{X}_{2}: \mathrm{ACN} /$ water ratio, $\mathrm{X}_{3}: \mathrm{pH}, \mathrm{X}_{4}:$ Temperature, Y: Extraction efficiency)

\begin{tabular}{|c|c|c|c|c|c|}
\hline Run order & $\mathrm{X}_{1}$ & $\mathrm{X}_{2}$ & $\mathrm{X}_{3}$ & $\mathrm{X}_{4}$ & $\mathrm{Y}$ \\
\hline 1 & 160 & 1 & 8 & 4 & $91.31 \pm 1.14$ \\
\hline 2 & 200 & 1.75 & 10 & 12 & $61.97 \pm 3.47$ \\
\hline 3 & 200 & 1.75 & 8 & 20 & $62.41 \pm 6.27$ \\
\hline 4 & 160 & 2.5 & 8 & 4 & $63.09 \pm 1.21$ \\
\hline 5 & 160 & 1 & 8 & 20 & $51.53 \pm 1.92$ \\
\hline 6 & 160 & 2.5 & 6 & 12 & $66.43 \pm 10.19$ \\
\hline 7 & 160 & 2.5 & 10 & 12 & $56.80 \pm 2.94$ \\
\hline 8 & 160 & 2.5 & 8 & 20 & $80.05 \pm 9.33$ \\
\hline 9 & 120 & 1 & 8 & 12 & $64.49 \pm 4.06$ \\
\hline 10 & 160 & 1.75 & 6 & 20 & $59.03 \pm 4.61$ \\
\hline 11 & 200 & 1.75 & 8 & 4 & $72.25 \pm 0.78$ \\
\hline 12 & 160 & 1.75 & 10 & 20 & $67.26 \pm 1.71$ \\
\hline 13 & 200 & 1 & 8 & 12 & $65.47 \pm 0.23$ \\
\hline 14 & 160 & 1 & 6 & 12 & $60.02 \pm 0.55$ \\
\hline 15 & 160 & 1.75 & 8 & 12 & $84.42 \pm 1.92$ \\
\hline 16 & 160 & 1.75 & 8 & 12 & $82.69 \pm 5.40$ \\
\hline 17 & 120 & 2.5 & 8 & 12 & $65.28 \pm 7.78$ \\
\hline 18 & 120 & 1.75 & 10 & 12 & $59.11 \pm 4.57$ \\
\hline 19 & 160 & 1.75 & 6 & 4 & $79.06 \pm 4.38$ \\
\hline 20 & 200 & 1.75 & 6 & 12 & $58.16 \pm 1.07$ \\
\hline 21 & 120 & 1.75 & 8 & 4 & $79.15 \pm 3.06$ \\
\hline 22 & 200 & 2.5 & 8 & 12 & $56.59 \pm 8.59$ \\
\hline 23 & 160 & 1.75 & 8 & 12 & $82.65 \pm 1.62$ \\
\hline 24 & 160 & 1.75 & 10 & 4 & $78.39 \pm 2.32$ \\
\hline 25 & 120 & 1.75 & 6 & 12 & $63.48 \pm 2.64$ \\
\hline 26 & 160 & 1 & 10 & 12 & $70.55 \pm 1.06$ \\
\hline 27 & 120 & 1.75 & 8 & 20 & $46.81 \pm 9.51$ \\
\hline
\end{tabular}

*Data is presented as the mean of duplicate analysis.

odology (RSM) (Minitab version 17). The high and low value for glucose $\left(\mathrm{X}_{1}\right)(120$ and $200 \mathrm{~g} / \mathrm{L}), \mathrm{ACN} /$ water ratio $\left(\mathrm{X}_{2}\right)$ (1 and 2.5), $\mathrm{pH}\left(\mathrm{X}_{3}\right)\left(6\right.$ and 10) and temperature $\left(\mathrm{X}_{4}\right)\left(4\right.$ and $\left.20^{\circ} \mathrm{C}\right)$ were selected for optimization and extraction efficiency $(\mathrm{Y})$ was obtained. Variable levels for glucose, $\mathrm{ACN} /$ water ratio, $\mathrm{pH}$, and temperature were selected according to the reported literature [22,24,37]. In the present study, three-level BBD was applied to investigate the effect of process conditions. For the optimization of operating conditions, 27 randomized experimental runs were conducted (Table 1). All the experiments were carried out in a $15 \mathrm{~mL}$ graduated glass tube.

The relationship of the independent variables and response was calculated by following second-order polynomial:

$$
\mathrm{Y}=\beta_{0}+\sum_{j=1}^{k} \beta_{j} \mathrm{x}_{j}+\sum_{j=1}^{k} \beta_{j j} \mathrm{x}_{j}^{2}+\sum_{i} \sum_{<j=2}^{k} \beta_{i j} \mathrm{x}_{i} \mathrm{x}_{j}+\mathrm{e}_{i}
$$

where $\mathrm{Y}$ is predicted response, $\mathrm{x}_{i}$ and $\mathrm{x}_{j}$ are variables, $\beta_{0}, \beta_{j}, \beta_{j j}$ and $\beta_{i j}$ are the regression coefficients for intercept, linear, square and interaction terms, respectively, and $\mathrm{e}_{i}$ is the error [38].

5. Effect of Specific Media Component and Simulated Broth on Sugaring Out Extraction of Erythromycin

The effect of specific media components (starch, soybean flour, 
Table 2. Simulated broth composition

\begin{tabular}{lcccc}
\hline \hline \multirow{2}{*}{ Compounds } & \multicolumn{4}{c}{ Composition $(\mathrm{g} / \mathrm{L})$} \\
\cline { 2 - 5 } & SFB1 & SFB2 & SFB3 & SFB4 \\
\hline Starch & 0 & 10 & 25 & 50 \\
Soybean flour & 0 & 5 & 15 & 27 \\
$\mathrm{NaCl}$ & 0 & 0.5 & 1 & 2 \\
$\mathrm{CaCO}_{3}$ & 0 & 1 & 3 & 5 \\
$\left(\mathrm{NH}_{4}\right)_{2} \mathrm{SO}_{4}$ & 0 & 2 & 4 & 5.52 \\
Erythromycin & 10 & 10 & 10 & 10 \\
\hline
\end{tabular}

$\mathrm{CaCO}_{3}, \mathrm{NaCl}$, and $\left.\left(\mathrm{NH}_{4}\right)_{2} \mathrm{SO}_{4}\right)$ on sugaring out extraction of erythromycin was studied. The upper limit of the media component was selected based on its concentration in the media. Each media component was added in an aqueous glucose solution and erythromycin was extracted in ACN-rich phase. The combined effect of media components was also studied by carrying out erythromycin extraction from simulated fermentation broth (SFB). SFB was prepared for four different concentrations of media components (Table 2). Extraction studies were carried out at the conditions optimized earlier.

\section{Erythromycin Fermentation and Extraction from the Real Fermentation Broth}

The cryopreserved culture of Saccharopolyspora erythrea was revived using growth media as prescribed by the culture bank. The growth medium consisted of the following components $(\mathrm{g} / \mathrm{L})$ : Beef extract 12 , peptone 2 , yeast extract 2 , tryptose 2 , calcium carbonate 0.1 , starch 0.1 , glucose 10 , agar 15 , cobalt chloride and ferric ammonium citrate in traces. For the revival, the culture was incubated at $30^{\circ} \mathrm{C}$ for 4 days. $10 \%(\mathrm{v} / \mathrm{v})$ of the above seed culture was inoculated in the optimized fermentation medium. Fermentation media composition was as follows $(\mathrm{g} / \mathrm{L})$ : starch 50 , soybean flour 27, $\mathrm{CaCO}_{3} 5, \mathrm{NaCl} 2$ and $\left(\mathrm{NH}_{4}\right)_{2} \mathrm{SO}_{4} 5.57$ [39]. Fermentation was carried out at $32{ }^{\circ} \mathrm{C}$ and $220 \mathrm{rpm}$ for 9 days. Fermentation broth obtained at the end of fermentation was used, as it is for erythromycin extraction. Also, extraction studies were carried out with fermentation broth after removal of biomass and other suspended solids to see the effect of suspended solids on erythromycin extraction. Suspended solids were removed by centrifugation at $4,000 \mathrm{~g}$ for 10 minutes. Supernatant collected was used for the sugaringout extraction of erythromycin [8]. Optimized conditions were used for extraction in both cases.

\section{Analytical Procedure}

The sample was analyzed by using a UV-visible spectrophotometer (Agilent Technologies). The sample was diluted with methanol and distilled water mixture $(1: 1)$ followed by the addition of phosphoric acid to the above mixture in a $2: 3$ ratio. The solution was heated in a boiling water bath for 20 minutes. The absorbance was measured at $485 \mathrm{~nm}$ [40]. Extraction efficiency and partition coefficient were calculated as follows:

$$
\begin{aligned}
& \% \text { Extraction efficiency }(\mathrm{w} / \mathrm{w}) \\
& =\frac{\text { Amount of erythromycin in upper phase }}{\text { Total amount of erythromycin }} \times 100
\end{aligned}
$$

Partition coefficient

$$
=\frac{\text { Concentration of erythromycin in upper phase }}{\text { Concentration of erythromycin in lower phase }}
$$

\section{Evaluation of Antimicrobial Activity}

Antimicrobial activity of erythromycin extracted using sugaringout from simulated and real broth was tested against B. pumilus and compared with standard erythromycin. Antimicrobial activity was tested using agar well diffusion method on nutrient agar medium in a petri plate. Agar plates were inoculated using the spread plate method by spreading bacterial strain under aseptic conditions. A $6 \mathrm{~mm}$ diameter well was punched in agar plate using a sterile tip. This was followed by the filling of well with $15 \mu \mathrm{L}$ of antibiotic solution. The inhibition zone of erythromycin surrounding the well was noted after $24 \mathrm{~h}$ of incubation [41]. Since the extracted erythromycin solution had ACN, the effect of ACN on the inhibition zone was also studied. The diameter of the inhibition zone was measured to quantify the antimicrobial activity of erythromycin.

\section{RESULTS AND DISCUSSION}

\section{Optimization of Process Parameters: Box Behnken Analysis}

BBD provides more efficient matrices for a large number of variables compared with CCD, hence, BBD was chosen for the optimization of process parameters for erythromycin extraction [38]. The response of BBD was measured in terms of extraction efficiency (Y). Experimental results of 27 randomized runs obtained under different conditions are shown in Table 1. The maximum extraction efficiency of $91.3 \%(\mathrm{w} / \mathrm{w})$ was obtained for the experimental run at the following process conditions: glucose: $160 \mathrm{~g} / \mathrm{L}$, ACN/water ratio: $1, \mathrm{pH}: 8$, and Temperature: $4^{\circ} \mathrm{C}$.

After applying the multiple regression analysis, the final predictive second order equation to achieve maximum extraction efficiency was obtained.

Table 3. Estimated coefficients for extraction efficiency

\begin{tabular}{lrrrrc}
\hline \hline Term & DF & Adj SS & Adj MS & F-value & P-value \\
\hline Model & 14 & $3,068.75$ & 219.19 & 19.70 & 0.000 \\
$\mathrm{X}_{1}$ & 1 & 0.18 & 0.18 & 0.02 & 0.900 \\
$\mathrm{X}_{2}$ & 1 & 19.07 & 19.07 & 1.71 & 0.215 \\
$\mathrm{X}_{3}$ & 1 & 5.22 & 5.22 & 0.47 & 0.506 \\
$\mathrm{X}_{4}$ & 1 & 770.30 & 770.29 & 69.22 & 0.000 \\
$\mathrm{X}_{1}{ }^{2}$ & 1 & 902.55 & 902.54 & 81.11 & 0.000 \\
$\mathrm{X}_{2}{ }^{2}$ & 1 & 381.14 & 381.13 & 34.25 & 0.000 \\
$\mathrm{X}_{3}{ }^{2}$ & 1 & 519.70 & 519.69 & 46.70 & 0.000 \\
$\mathrm{X}_{4}{ }^{2}$ & 1 & 69.66 & 69.65 & 6.26 & 0.028 \\
$\mathrm{X}_{1} \times \mathrm{X}_{2}$ & 1 & 23.36 & 23.36 & 2.10 & 0.173 \\
$\mathrm{X}_{1} \times \mathrm{X}_{3}$ & 1 & 16.76 & 16.75 & 1.51 & 0.243 \\
$\mathrm{X}_{1} \times \mathrm{X}_{4}$ & 1 & 126.54 & 126.53 & 11.37 & 0.006 \\
$\mathrm{X}_{2} \times \mathrm{X}_{3}$ & 1 & 101.62 & 101.62 & 9.13 & 0.011 \\
$\mathrm{X}_{2} \times \mathrm{X}_{4}$ & 1 & 804.77 & 804.77 & 72.32 & 0.000 \\
$\mathrm{X}_{3} \times \mathrm{X}_{4}$ & 1 & 19.81 & 19.81 & 1.78 & 0.207 \\
$\mathrm{R}^{2}$ & & & $96 \%$ & & \\
\hline
\end{tabular}




$$
\begin{aligned}
\mathrm{Y}= & -264.3+2.32 \mathrm{X}_{1}+62.3 \mathrm{X}_{2}+39.93 \mathrm{X}_{3}+(-7.71) \mathrm{X}_{4}+(-0.008) \mathrm{X}_{1}^{2} \\
& +(-15.03) \mathrm{X}_{2}^{2}+(-2.46) \mathrm{X}_{3}^{2}+(-0.056) \mathrm{X}_{4}^{2}+(-0.80) \mathrm{X}_{1} \mathrm{X}_{2}+0.025 \mathrm{X}_{1} \mathrm{X}_{3} \\
& +0.01 \mathrm{X}_{1} \mathrm{X}_{4}+\left(-3.36 \mathrm{X}_{2} \mathrm{X}_{3}\right)+2.36 \mathrm{X}_{2} \mathrm{X}_{4}+0.139 \mathrm{X}_{3} \mathrm{X}_{4}
\end{aligned}
$$

where Y- Extraction efficiency, $\mathrm{X}_{1}$ - Glucose concentration, $\mathrm{X}_{2}$ ACN/Water ratio, $\mathrm{X}_{3}-\mathrm{pH}, \mathrm{X}_{4}$ - Temperature

Analysis of variance (ANOVA) was carried out to analyze the significance of the model (Table 3). Key factors that showed the significance of variables were analyzed using ANOVA. $\mathrm{R}^{2}$ values near 1 indicate a better response, which showed that the model fits well [42]. The regression coefficient $\left(\mathrm{R}^{2}\right)$ for extraction efficiency was $96 \%$, which shows only $4 \%$ variation was not explained by the model. Also, a high value of adjusted $\mathrm{R}^{2}(91 \%)$ indicates the high significance of the model. The significance of the coefficient was determined using p-value and F-value. When the magnitude of the p-value is smaller and the magnitude of the F-value is higher, the coefficient will be more significant [42]. Smaller the magnitude of the $p$-value $(p<0.05)$, the more significant the effect of a variable on response. Analysis of variance of the regression model indicated that the model is highly significant, as it marked a high Fvalue (19.70) with a very low p-value (0.000). Insignificant lack of fit $(\mathrm{p}<0.05)$ was also a sign of the good adequacy of the model.

Temperature was found to be the most important parameter for the recovery of erythromycin using sugaring-out extraction. Even though the effect of glucose, ACN/water ratio, and $\mathrm{pH}$ was not that significant, those were also important to enable the recovery of erythromycin from the aqueous phase. The amount of erythromycin recovered from the aqueous phase would be insignificant without either one of the parameters. Contour plots showing the interaction among different variables and extraction efficiency are shown in Fig. 1. Each contour plot has a middle variable held constant to investigate the effect of other variables.

1-1. Effect of Glucose Concentration and ACN/Water Ratio on Sugaring-out Extraction of Erythromycin

It was observed that erythromycin extraction efficiency increased from $60 \%$ to $80 \%(\mathrm{w} / \mathrm{w})$ with an increase in glucose concentration (Fig. 1(a)). High glucose concentration favors phase separation, which facilitates the maximum recovery of erythromycin from the aqueous phase. An increase in extraction efficiency could be attributed to the formation of a hydrogen bond between glucose and water. The addition of glucose replaces the existing ACN-water hydrogen bond, thus pushing ACN molecules out of water. Hence, an increase in glucose concentration replaces more hydrogen bonds between water and ACN, leading to separation of ACN [24]. Since erythromycin is hydrophobic, it moves to the solvent-rich phase, consequently enhancing the extraction of erythromycin.

Square interaction of ACN/water ratio and two-way interaction with $\mathrm{pH}$ and temperature had a significant effect. The extraction efficiency of erythromycin was optimum at the ACN/water volume ratio of 1.2 to 2.1. Maximum erythromycin extraction in this region could be attributed to the microheterogeneity of the ACNwater solution. Microheterogeneity in ACN-water solution is observed between acetonitrile mole fraction of 0.2-0.8. In this range (1.2-2.1), the ACN-water solution contains clusters of ACN molecules attached with water molecules by a hydrogen bond. Since erythromycin is a hydrophobic molecule, this leads to the transfer

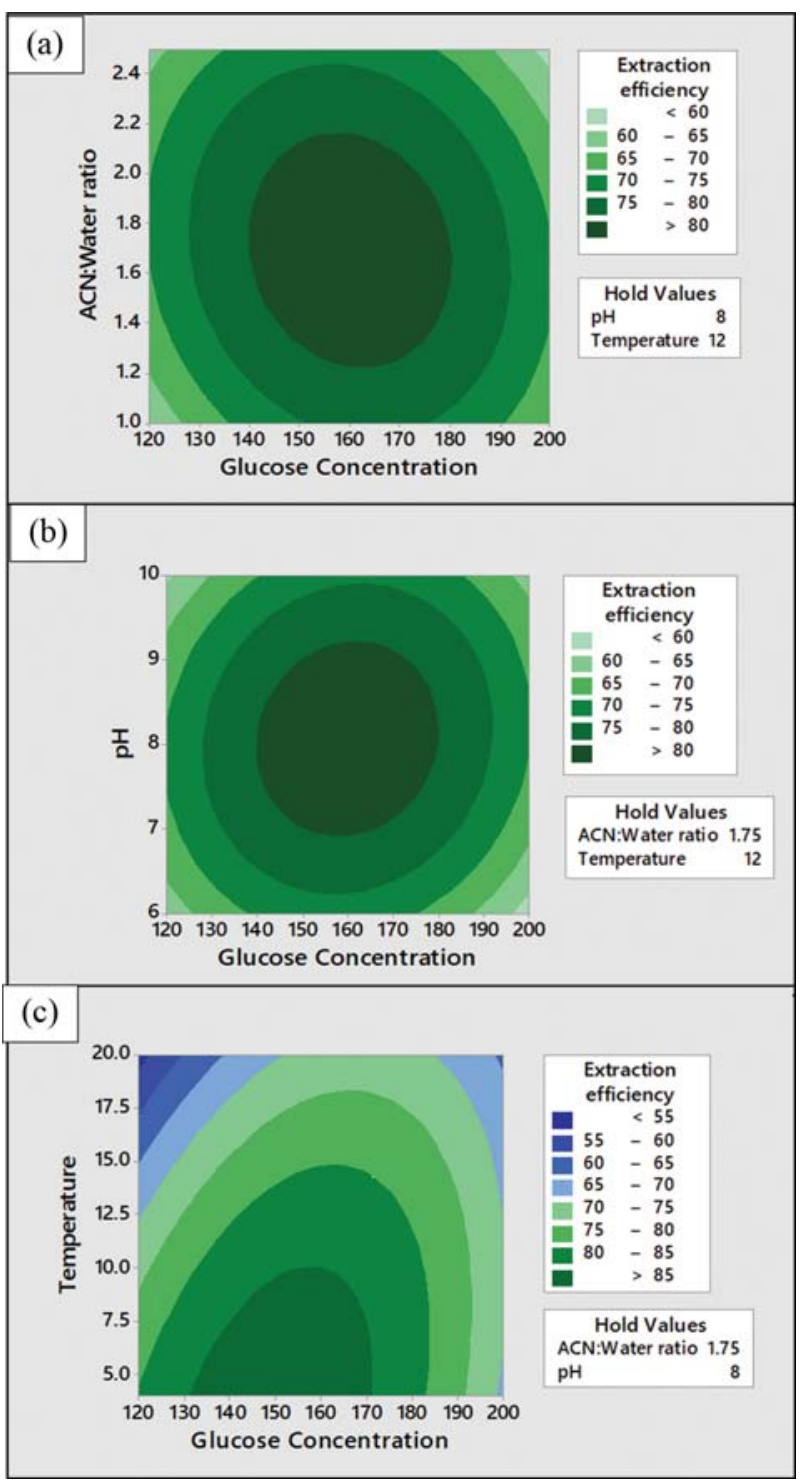

Fig. 1. Contour plots for extraction efficiency: Simultaneous effect of glucose concentration and (a) $\mathrm{ACN} /$ water ratio (b) $\mathrm{pH}$ and (c) temperature (Initial erythromycin concentration=10 g/L).

of erythromycin from aqueous solution to the solvent-rich phase, consequently into better extraction efficiency. ACN mole fraction between 0.8 to 1.0 makes the solution more homogeneous and, hence, does not result in phase separation [43].

1-2. Effect of $\mathrm{pH}$ and Temperature on Sugaring Out Extraction of Erythromycin

ANOVA analysis showed that the interactive effect of $\mathrm{pH}$ was significant (Table 3). Fig. 1(b) shows that erythromycin extraction was optimum between $\mathrm{pH} 7$ to 8.5. Erythromycin is capable of undergoing protonation as it is a weak base containing one tertiary amine group (pKa is approximately 8.18). It indicates that the extraction of erythromycin can be carried out using electrostatic interaction. Erythromycin gains positive charge when $\mathrm{pH}<\mathrm{pKa}$ as $[\mathrm{ErH}]^{+}$species dominates [44]. It may cause strong electrostatic interaction between $\mathrm{ACN}$ which has nitrogen that carries a partial negative charge and erythromycin having a positive charge. This 
would enhance erythromycin recovery. It demonstrated that a basic environment $(\mathrm{pH}>7)$ enables maximum recovery of erythromycin. Thus, maximum extraction is obtained in the alkaline range which supports the theory [14].

Statistical analysis (Table 3) showed that temperature was the most dominating factor for extraction efficiency. Fig. 1(c) shows that maximum erythromycin was extracted from aqueous solution in the temperature range of $4-8^{\circ} \mathrm{C}$. It was identified that erythromycin extraction was maximum at low temperature, as it favors better phase separation between ACN and water, which led to improved recovery of erythromycin. Phase separation mainly depends on the degree of hydrogen bonding between water molecules. At lower temperature, water molecules become more organized and tend to come closer. Due to this, ACN molecules find less chance to replace water molecules. At low temperature, more ACN molecules are pushed out from the lower phase, which leads to better phase separation [24] and higher extraction efficiency.

\section{1-3. Validation of Predicted Optimized Condition}

At the predicted optimum conditions (glucose $156.3 \mathrm{~g} / \mathrm{L}, \mathrm{ACN} /$ water ratio $1, \mathrm{pH} 8.3$, and temperature $4{ }^{\circ} \mathrm{C}$ ), the predicted response by the model for the optimum performance was 14.6 , and $88.8 \%$ (w/w) for partition coefficient and erythromycin extraction, respectively. When experiments were carried out at the predicted optimum conditions, it resulted in a partition coefficient of 13.5 and erythromycin extraction efficiency of $95.1 \%(w / w)$. These results revealed that the experimental response was in good agreement with a predicted response. This further confirms that BBD design was successfully used for the optimization of process parameters for the extraction of erythromycin using sugaring out.

All the above studies were carried out by incubating the aqueous erythromycin solution in a water bath chiller for $4 \mathrm{~h}$. Hence, the effect of time on sugaring out extraction was studied (Fig. 2). It was observed that the maximum erythromycin extraction was achieved in $1 \mathrm{~h}$. Thus, as compared to butyl acetate (35 h), erythromycin required less time for two-phase formation.

\section{Effect of Specific Fermentation Media Component on Sug-} aring Out Extraction of Erythromycin

The effect of media components on sugaring out extraction was

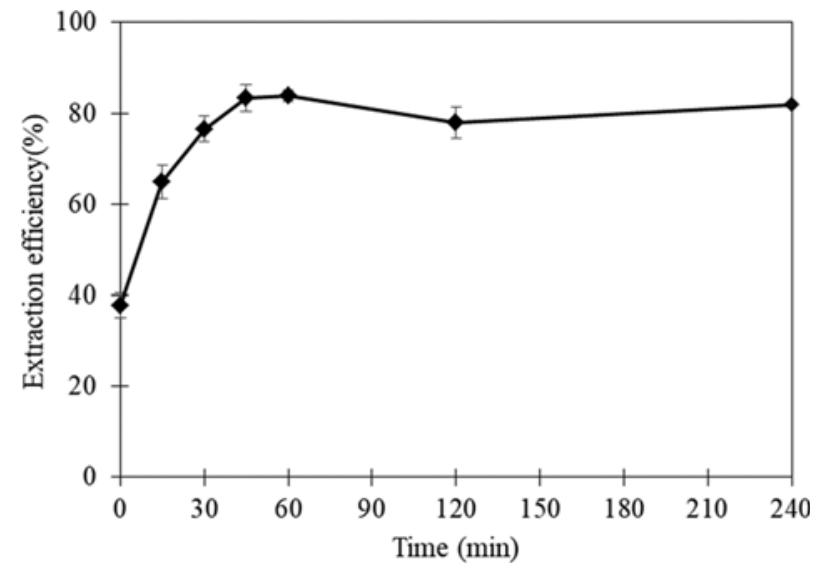

Fig. 2. Extraction of erythromycin using sugaring-out: Effect of time (Glucose 156.3 g/L, ACN/Water ratio 1, Temperature $4^{\circ} \mathrm{C}$ and pH 8.3). studied at conditions optimized earlier (glucose $156.3 \mathrm{~g} / \mathrm{L}$, temperature $4{ }^{\circ} \mathrm{C}, \mathrm{ACN} /$ water ratio 1 , and $\left.\mathrm{pH} 8.3\right)$. It was observed that increasing concentration of starch $(0-50 \mathrm{~g} / \mathrm{L})$, soybean flour $(0-27$ $\mathrm{g} / \mathrm{L})$, and calcium carbonate $(0-5 \mathrm{~g} / \mathrm{L})$ showed a significant decrease in erythromycin extraction (starch 90.6-77.9\%; soybean flour 90.659.4\%; $\mathrm{CaCO}_{3} 85-65 \%$ ) (Fig. 3). At a high concentration of starch and soybean flour, the low solubility of starch and soybean flour may have indirectly affected the diffusion behavior of erythromycin. A high amount of starch and soybean flour would form a viscous solution leading to lower extraction efficiencies. Ammonium sulfate $(0-5.5 \mathrm{~g} / \mathrm{L})$ and sodium chloride $(0-2 \mathrm{~g} / \mathrm{L})$ had an insignificant effect on sugaring-out extraction of erythromycin. Sodium chloride and ammonium sulfate, which can act as a mass separating agent, did not affect the extraction of erythromycin as it is present in a very small concentration in the media.

3. Sugaring-out Extraction of Erythromycin from Simulated Fermentation Broth (SFB) and Real Fermentation Broth (RFB)

Erythromycin was extracted from a simulated broth of varying composition. The composition of simulated broth was varied assuming that some of the components are unutilized at the end of fermentation. This study also revealed the combined effect of media
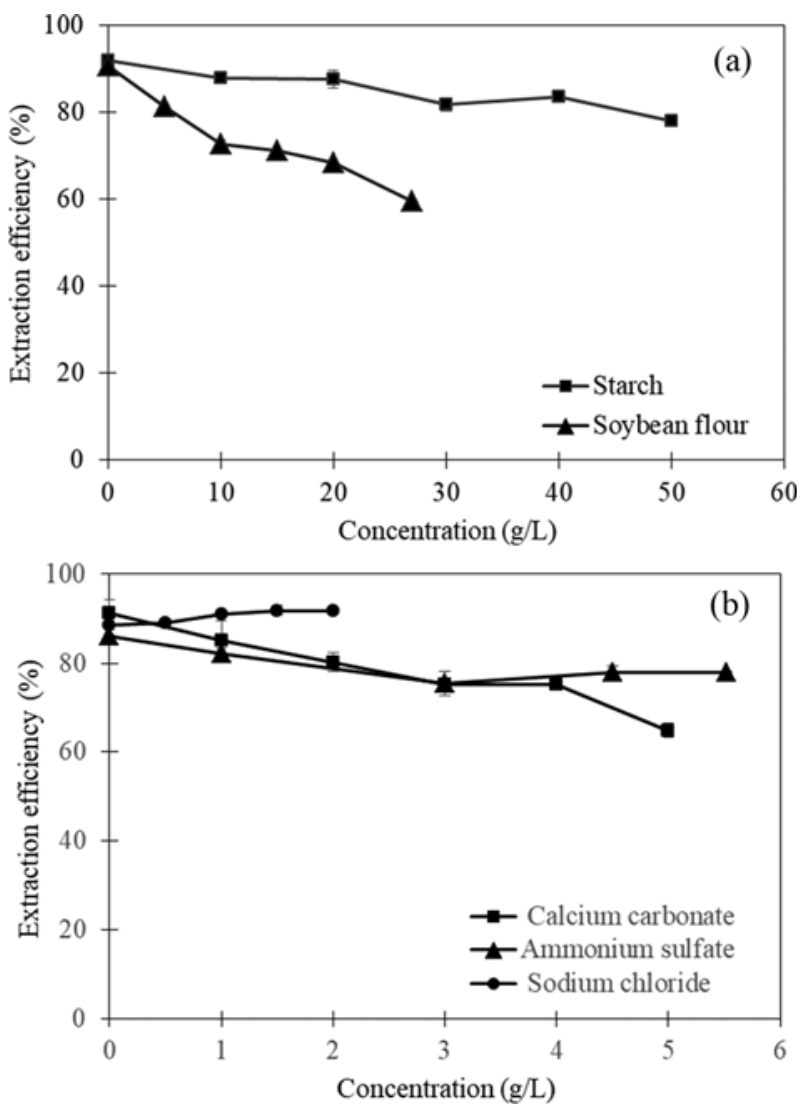

Fig. 3. Effect of specific media component on erythromycin extraction (a) soyabean flour and starch flour (b) Calcium carbonate; ammonium sulfate and sodium chloride (Initial erythromycin $10 \mathrm{~g} / \mathrm{L}$, Glucose $156.3 \mathrm{~g} / \mathrm{L}$, ACN/Water ratio 1, Temperature $4^{\circ} \mathrm{C}$ and $\mathrm{pH}$ 8.3). (Maximum concentration of a media component was chosen based on its initial concentration in the media.). 


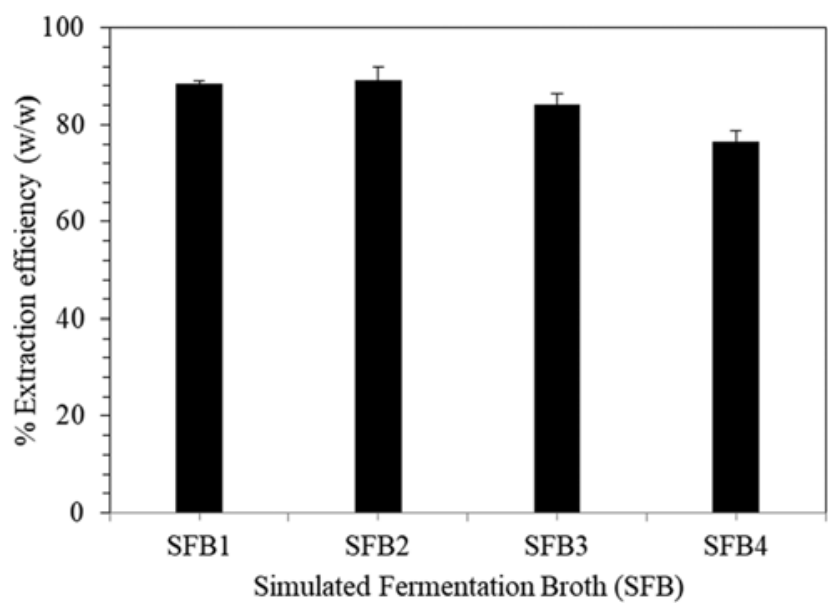

Fig. 4. The combined effect of media components in the form of simulated fermentation broth (SFB). The detailed composition of each media corresponding to SFB1, SFB2, SFB3, and SFB4 is included in Table 2 (Sugaring out conditions: Glucose $156.3 \mathrm{~g} / \mathrm{L}, \mathrm{ACN} /$ Water ratio 1 , Temperature $4^{\circ} \mathrm{C}$ and pH 8.3).

component on erythromycin extraction. For the simulated broth study, the concentration of fermentation broth in three range was chosen for sugaring out extraction of erythromycin. Fig. 4 shows the extraction efficiency of erythromycin using optimized conditions for a selected composition of simulated broth. At a high concentration of media components in a broth, extraction efficiency decreased (from 89 to $76 \% \mathrm{w} / \mathrm{w}$ ). The high concentration of media components was responsible for declined partition behavior of erythromycin. At high media components, undissolved media components mainly starch and soybean flour may act as an obstacle in erythromycin extraction.

The extraction of erythromycin from real fermentation broth was also carried out using earlier optimized conditions. Erythromycin titer obtained through fermentation was $6.5 \pm 0.2 \mathrm{~g} / \mathrm{L}$. The same broth was used for the sugaring-out extraction of erythromycin.

From simulated broth studies, it was observed that a high concentration of media components had a negative effect on the extraction of erythromycin from the broth. Considering this, erythromycin was extracted from the real broth as well as the simulated broth under two different conditions. Suspended solids were removed, using centrifugation, prior to sugaring-out extraction of erythromycin from broth. Removal of suspended solids enhanced the extraction efficiency from $76.4 \%$ to $85.8 \%(\mathrm{w} / \mathrm{w})$ and $72.1 \%$ to $86.6 \%$ for simulated and real broth, respectively (Fig. 5). Also, erythromycin extraction/extraction efficiency from simulated and real broth was almost unchanged. Presence of fermentation byproducts or impurities did not affect the extraction efficiency. Thus, erythromycin was successfully extracted from real fermentation broth using sugaring-out.

Salting-out extraction of erythromycin was also studied at the optimum conditions reported earlier [14]. This resulted in $94 \%$ $(\mathrm{w} / \mathrm{w})$ extraction of erythromycin at $25^{\circ} \mathrm{C}$ and $\mathrm{pH} 10$ with $10 \%$ (w/w) $\mathrm{NaCl}$ and $\mathrm{ACN} /$ water ratio of 1 . Though the extraction efficiency in the case of salting-out was higher, one needs to recover

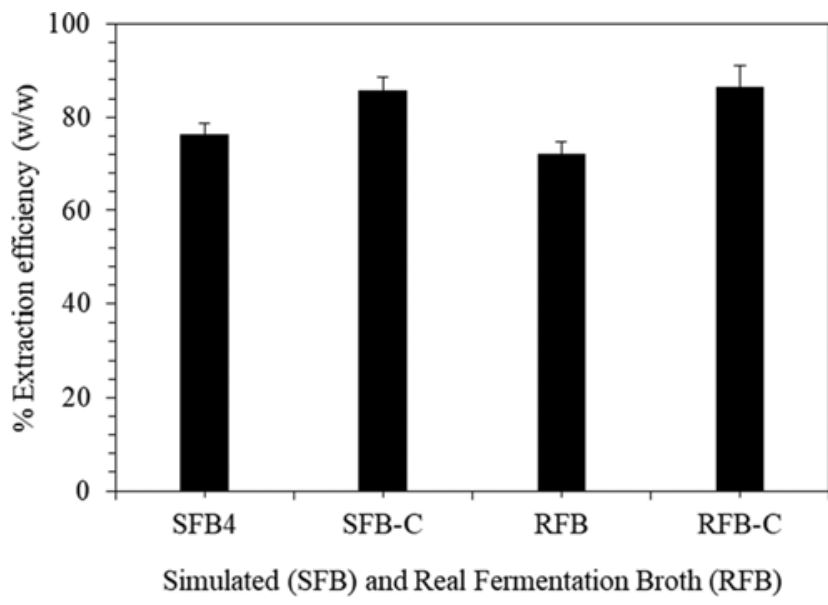

Fig. 5. Erythromycin extraction from simulated (SFB) and real fermentation broth (RFB): Effect of suspended matter. SFB4 composition included in Table 2. SFB4 and RFB were not centrifuged. C-broth was centrifuged before sugaring out (Sugaring out extraction conditions: Glucose $156.3 \mathrm{~g} / \mathrm{L}, \mathrm{ACN} /$ Water ratio 1 , Temperature $4^{\circ} \mathrm{C}$ and $\mathrm{pH}$ 8.3.).

salts from the bottom phase. In the case of sugaring-out extraction, the bottom phase (containing sugar) can be used as a base for the fermentation medium. Recent studies on sugaring out extraction of lactic acid [26], acetoin [27] and 2,3 butanediol [25] have used the bottom phase for fermentation. The performance of the bottom phase-based fermentation media and fresh media was comparable. Thus, sugaring out overcomes one of the major problems of salting-out extraction.

Another study reported sugaring-out extraction of erythromycin from aqueous solution with a maximum partition coefficient of 1.62 and 2.31 at $25^{\circ} \mathrm{C}$ with fructose (15 wt\%) and maltose (11 wt $\%)$, respectively [30]. It also reports a higher partition coefficient of 4.7 with sorbitol (sugar alcohol). In the present work, a partition coefficient of $11.5 \pm 0.5$ was obtained for simulated as well as a real broth with $15.3 \%(\mathrm{w} / \mathrm{w})$ glucose at $4{ }^{\circ} \mathrm{C}$ and $\mathrm{ACN} /$ water ratio of 1 ( $\mathrm{pH} 8.3$ ). Thus, the present work reports higher partitioning (4-5 times) than reported methods employing carbohydrates as a mass separating agent.

\section{Antimicrobial Activity of Extracted Erythromycin}

One of the important aspects of the separation of the biologically derived product is its qualitative analysis. Based on the authors' review of the literature, there are no studies directed for qualitative assay to examine whether the used extraction system affected the antimicrobial activity of erythromycin. Antimicrobial activity of sugaring out extracted erythromycin was measured in terms of the diameter of the inhibition zone. Since erythromycin is extracted into $\mathrm{ACN}$, the inhibition zone was studied for $\mathrm{ACN}$ as well. Standard erythromycin dissolved in ACN was used as a control. It can be seen from Fig. 6 that there was no inhibition zone for pure can, that is, the added amount of ACN did not inhibit the growth of bacteria. In the case of control, the diameter of the inhibition zone was $35 \mathrm{~mm}$. The diameter of the inhibition zone for erythromycin extracted using sugaring out from simulated and real fermentation broth was $33 \mathrm{~mm}$ and $35 \mathrm{~mm}$, respectively. The addition of 

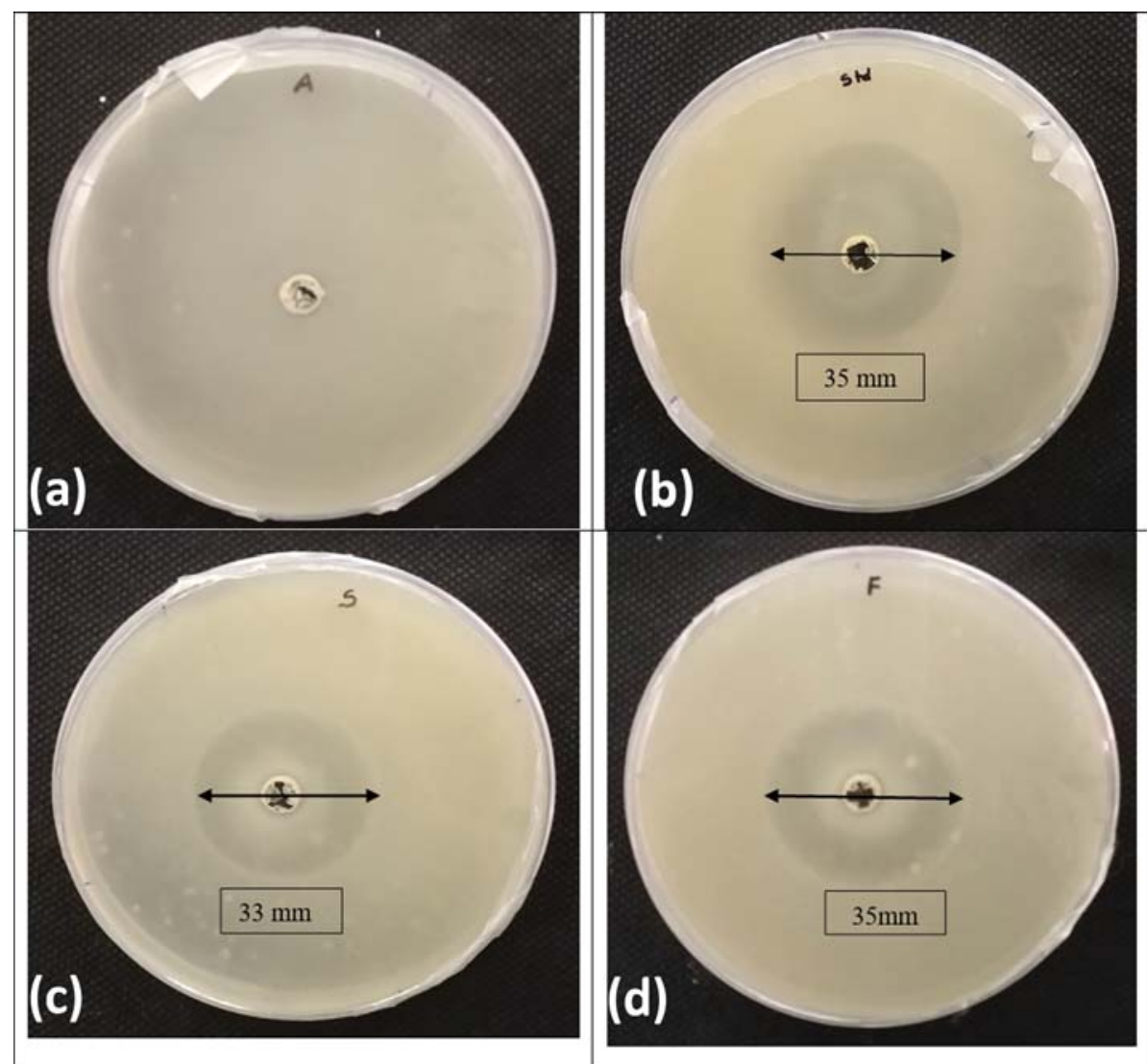

Fig. 6. Antimicrobial properties of erythromycin obtained (a) Pure ACN, (b) ACN+standard erythromycin, (c) Erythromycin extracted in $\mathrm{ACN}$ from the simulated broth using sugaring-out, (d) Erythromycin extracted in ACN from the real broth using sugaring out.

erythromycin inhibits or kills the bacteria. This leads to a zone where no bacterial growth is found. Larger size or diameter of the inhibition zone would imply the strength, in terms of antimicrobial activity, of erythromycin. The diameter of inhibition zone of control (i.e., standard erythromycin) was comparable to that of erythromycin extracted using sugaring out. Thus, sugaring out extraction not only separated erythromycin efficiently but also retained its antimicrobial activity.

\section{CONCLUSIONS}

The feasibility of using sugaring out for extraction of erythromycin was investigated. The extraction efficiency of $88.8 \%(\mathrm{w} / \mathrm{w})$ was obtained for a model system at the optimized conditions of glucose $156.3 \mathrm{~g} / \mathrm{L}, \mathrm{ACN} /$ water ratio $1, \mathrm{pH} 8.3$ and temperature $4{ }^{\circ} \mathrm{C}$. Equilibrium study showed that sugaring out was rapid and equilibrium was attained in less than $1 \mathrm{~h}$. Effect of media components (single and combined) showed that high concentration of starch, soybean flour, and $\mathrm{CaCO}_{3}$ affected the sugaring-out extraction of erythromycin. Combined media components had a pronounced effect only at higher concentration. However, the removal of suspended solids from broth enhanced the sugaring-out extraction of erythromycin. Extraction efficiency of $85.8 \%(w / w)$ and $86.6 \%(w / w)$ was achieved for simulated and real fermentation broth, respectively. Though salting out yielded $94 \%(\mathrm{w} / \mathrm{w})$ extraction efficiency, it suffered from a major drawback of salt recovery. The partition coefficient obtained in the present work is 4-5 times higher than other methods using carbohydrates as a mass separating agent. Further, the antimicrobial properties of erythromycin were retained even after sugaring out. Thus, sugaring out yields high efficiency while retaining the activity of the molecule. Further work will focus on the separation of erythromycin from the solvent rich phase and recovery of solvent and sugars.

\section{ACKNOWLEDGEMENT}

Dr. Pradip B. Dhamole would like to thank Science and Engineering Research Board (SERB)-Department of Science and Technology (DST) (Govt. of India) for funding this work (vide Sanction order No. EMR/2016/005672 dated September 13, 2017).

\section{REFERENCES}

1. A. P. Anisimov and K. K. Amoako, J. Med. Microbiol., 55, 1461 (2006).

2. F. Bouzid, H. Astier, D. A. Osman, E. Javelle, M. O. Hassan, F. Simon, E. Garnotel and M. Drancourt, Int. J. Antimicrob. Agents, 51, 235 (2018).

3. J. C. Hoyt and R. A. Robbins, FEMS Microbiol. Lett., 205, 1 (2001).

4. P. Gautret, J. Lagier, P. Parola, B. Doudier, J. Courjon, B. La Scola, 
J. Rolain, P. Brouqui, D. Raoult, M. Mailhe, B. Doudier and J. Courjon, Int. J. Antimicrob. Agents, 56, 105949 (2020).

5. H. Ulrich and M. M. Pillat, Stem Cell Rev Rep, 16, 434 (2020).

6. J. Liu, Y. Chen, W. Wang, M. Ren, P. Wu, Y. Wang, C. Li, L. Zhang, H. Wu, D. T. Weaver and B. Zhang, Metab. Eng., 39, 29 (2017).

7. G. V. Sanghvi, D. Ghevariya, S. Gosai, R. Langa, N. Dhaduk, P. D. Kunjadia, D. J. Vaishnav and G. S. Dave, Biotechnol. Reports, 1-2, 2 (2014).

8. X. Zou, H. F. Hang, J. Chu, Y. P. Zhuang and S. L. Zhang, Bioresour. Technol., 100, 3358 (2009).

9. S. N. M. Aziz, A. W. Zularisam and A. M. M. Sakinah, Sep. Purif. Technol., 229, 115816 (2019).

10. Z. Li, F. Qin, H. Bao and X. Gu, J. Chem. Technol. Biotechnol., 80, 772 (2005).

11. M. H. L. Ribeiro and I. A. C. Ribeiro, Sep. Purif. Technol., 45, 232 (2005).

12. X. D. Weng, Y. L. Ji, R. Ma, F. Y. Zhao, Q. F. An and C. J. Gao, J. Membr. Sci., 510, 122 (2016).

13. Y. He, G. Chen, Z. Ji and S. Li, Sep. Purif. Technol., 66, 390 (2009).

14. Q. Le, L. Shong and Y. Shi, Sep. Purif. Technol., 24, 85 (2001).

15. S. C. Lee, J. Ind. Eng. Chem., 15, 403 (2009).

16. A. M. A. Nabais and J. P. Cardoso, Bioprocess Eng., 21, 157 (1999).

17. A. M. A. Nabais and J. P. Cardoso, Bioprocess Eng., 13, 215 (1995).

18. E. N. Lightfoot and J.S. Moscariello, Bioseparations, Biotechnol. Bioeng., 87, 259 (2004).

19. L. A. Jones, J. B. Prabel, J. J. Glennon, M. F. Copeland and R. J. Kavlock, J. Agric. Food Chem., 41, 735 (1993).

20. H. Leinonen, Corrosion, 52, 337 (1996).

21. Y. Gu and P. H. Shih, Enzyme Microb. Technol., 35, 592 (2004).

22. B. Wang, T. Ezejias, H. Feng and H. Blaschek, Chem. Eng. Sci., 63, 2595 (2008).

23. B. Wang, H. Feng, T. Ezeji and H. Blaschek, Chem. Eng. Technol., 31, 1869 (2008).

24. P. B. Dhamole, P. Mahajan and H. Feng, J. Chem. Eng. Data, 55, 3803 (2010).

25. J. Y. Dai, C. J. Liu and Z. L. Xiu, Process Biochem., 50, 1951 (2015).
26. L. Yan, Y. Q. Sun and Z. L. Xiu, Sep. Purif. Technol., 161, 152 (2016). 27. J. Y. Dai, L. H. Ma, Z. F. Wang, W. T. Guan and Z. L. Xiu, Bioprocess Biosyst. Eng., 40, 423 (2017).

28. Y. Sun, S. Zhang, X. Zhang, Y. Zheng, Z. Xiu, S. Zhang, X. Zhang, Y. Zheng and Z. Xiu, Sep. Purif. Technol., 204, 133 (2018).

29. Y. Sun, X. Zhang, Y. Zheng, L. Yan and Z. Xiu, Sep. Purif. Technol., 209, 972 (2019).

30. B. A. Shoushtari, G. Pazuki, J. R. Shahrouzi, S. Shahriari and N. Hadidi, Fluid Phase Equilib., 505, 112360 (2020).

31. F. Moradi and J.R. Shahrouzi, Fluid Phase Equilib., 507, 112388 (2020).

32. S. R. Chia, K. W. Chew, P. L. Show, M. Sivakumar, T. C. Ling and Y. Tao, J. Oceanol. Limnol., 37, 898 (2019).

33. G. d. B. Cardoso, I. N. Souza, M. M. Pereira, M. G. Freire, C. M. F. Soares and Á. S. Lima, Sep. Purif. Technol., 136, 74 (2014).

34. P. B. Dhamole, S. Chavan, R. G. Patil, H. Feng, M. Bule and P. Kinninge, Korean J. Chem. Eng., 33, 1860 (2016).

35. W. H. Tsai, H. Y. Chuang, H. H. Chen, Y. W. Wu, S. H. Cheng and T. C. Huang, J. Chromatogr. A, 1217, 7812 (2010).

36. Z. Zhu, Y. Zhang, J. Wang, X. Li, W. Wang and Z. Huang, J. Chromatogr. A, 1601, 104 (2019).

37. S. C. Chuo, N. Abd-Talib, S. H. Mohd-Setapar, H. Hassan, H. M. Nasir, A. Ahmad, D. Lokhat and G. M. Ashraf, Sci. Rep., 8, 1 (2018).

38. M. A. Bezerra, R. E. Santelli, E. P. Oliveira, L. S. Villar and L. A. Escaleira, Talanta, 76, 965 (2008).

39. X. Zou, H. feng Hang, J. Chu, Y. Ping Zhuang and S. Liang Zhang, Bioresour. Technol., 100, 1406 (2009).

40. Y. Zhu, D. Jiang, D. Sun, Y. Yan and C. Li, J. Environ. Chem. Eng., 4, 3570 (2016).

41. M. Balouiri, M. Sadiki and S. K. Ibnsouda, J. Pharm. Anal., 6, 71 (2016).

42. N. Anuar, A. F. M. Adnan, N. Saat, N. Aziz and R. M. Taha, Scientific World J., 2013, 810547 (2013).

43. S. Koley and S. Ghosh, ChemPhysChem., 16, 3518 (2015).

44. M. S. Manic, M. N. Da Ponte and V. Najdanovic-Visak, Chem. Eng. J., 171, 904 (2011). 